tron-neutral hydrogen bremsstrahlung (free-free emission) in envelopes surrounding stars of early type. They presented convincing fits to the infrared spectral energy distributions of a wide variety of early-type stars by assuming the superposition of a low temperature free-free emitting gas shell on an underlying stellar spectral energy distribution. Dyck and Milkey proceeded from purely theoretical considerations to estimate circumstellar envelope parameters. Typical values of temperature, neutral hydrogen density and sizes were derived to be $3,000 \mathrm{~K}, 10^{12}$ atoms $/ \mathrm{cm}^{3}$ and 100 1,000 times a typical stellar radius respectively.

The importance of gas emission in the interpretation of infrared excesses received further support from the report of a strong correlation between hydrogen line emission, Balmer continuum emission and observed flux in the infrared. This correlation was found by Drs S. E. Strom (Kitt Peak National Observatory) and L. V. Kuhi (Berkeley Astronomy Department) to apply to a wide variety of stars in the temperature range 4,000 to $20,000 \mathrm{~K}$.

There seems no doubt, however, that dust plays an important, although certainly not exclusive, part in the infrared emission properties of many objects at all spectral types. Dr E. P. Ney (University of Minnesota) presented an impressive array of high resolution observations of the so-called $11 \mu \mathrm{m}$ "bump" attributed by many authors to emission by silicate dust grains. Although this feature has been observed for some time in the infrared spectra of many oxygen-rich stars of late spectral type, $\mathrm{Dr} \mathrm{Ney}$ also presented evidence that the silicate feature occurs in a few of the early-type, hot stars in Orion I, the very young stellar association. The dust envelopes producing this feature appear to be cool (a few hundred degrees $\mathrm{K}$ ) and quite extended (angular size 20 arc seconds corresponding to a linear size of about $10^{17} \mathrm{~cm}$ ). It seems most likely that the dust surrounding these early-type stars is remnant material from the cloud out of which the star was formed.

Dr N. J. Woolf (University of Minnesota) also reported the observation of the $11 \mu \mathrm{m}$ silicate feature in absorption against the Becklin-Neugebauer source in Orion and against the infrared core of the galactic centre. An absorption feature at $3.1 \mu \mathrm{m}$ was also seen against the Orion source; if this feature is attributed to interstellar ice, the ice-tosilicate ratio is estimated to be between $1 / 6$ and $1 / 20$. Dr Woolf's observations suggest that silicate material is the principal constituent of the interstellar dust grains Whether all the silicates found in the interstellar medium have been created in the envelopes of late-type stars still seemed to be open to question. The

identification of the $11 \mu \mathrm{m}$ feature as a silicate signature received considerable support from a detailed laboratory analysis of the optical properties of almandine garnet by Dr D. R. Huffman and his associates (University of Arizona).

Dr J. E. Gaustad (Berkeley Astronomy Department) presented new, high resolution observations made with a Fourier transform spectrometer. His data revealed an emission feature at $12 \mu \mathrm{m}$ in several carbon-rich late-type stars which he attributed to $\mathrm{SiC}$. This identification is made plausible not only by the wavelength match but by the expected abundance of $\mathrm{SiC}$ as a condensate in the envelopes of carbon-rich stars.

Dr Strom reviewed the observed infrared properties of extremely young stellar objects. Infrared excesses are observed in young stars of all spectral types; these excesses seem to result from

\title{
Cholesterol and Catecholamine Uptake
}

A POSSIBLE rationale for the fashionable condemnation of high intakes of the steroid cholesterol as a causative factor in hypertension is hinted at by $\mathbf{P}$. $\mathbf{J}$. Salt and L. L. Iversen, from the University of Cambridge, in a report in next Wednesday's Nature New Biology (July 19). They show that cholesterol inhibits the extraneuronal uptake of catecholamines.

Catecholamine uptake, rigorously explored in the $1960 \mathrm{~s}$, occurs by two processes. In sympathetically-innervated organs, perfusion with exogenous radioactively-labelled catecholamines results in rapid labelling of the tissue. Iversen, using the isolated rat heart, perfused through the coronary vasculature, has found that with low concentrations of noradrenaline, most of the incorporated label entered postganglionic sympathetic nerve terminals; in hearts taken from animals lacking such terminals as a result of immunosympathectomy at birth, uptake was almost completely absent. But perfusion of rat hearts with substantially higher concentrations of catecholamines showed that, in addition to uptake by neuronal sites (termed uptake 1), transfer to cardiac muscle cells also took place, by a process (uptake 2) with properties clearly distinct from those of uptake 1. Thus, uptake 2, unlike the neuronal process, has a higher affinity for adrenaline than noradrenaline. Similarly, catecholamines entering extraneuronal sites are not firmly retained, whereas the uptake into terminals is followed by transfer to intraneuronal storage vesicles, and therefore cannot readily be reversed.

Physiologically, both uptake processes are important for preventing released catecholamines from continuing to act after their effect is complete. Noradrenaline is the transmitter released at a combination of free-free and dust radiation possibly from remnant material in circumstellar envelopes. Some combined infrared and optical spectroscopic evidence suggests that these envelopes are not spherical, but possibly disk-like; this view finds some support from the polarimetric data presented by Drs B. Zellner and K. Serkowski (University of Arizona). Many of the details of pre-main sequence stellar and envelope evolution, however, are not yet clear. The appearance of mass outflow in most young stars of all types runs counter to the hydrodynamical models of pre-main sequence evolution proposed by $\mathrm{Dr} \mathrm{R}$. Larson (Yale University) in which a stellar core gradually accretes material from the surrounding envelope. Considerably more effort could well be applied to the identification and spectroscopic study of stars younger than those found in young

adrenergic nerve terminals, and thus its recapture by the uptake 1 process ensures its removal from extracellular sites as well as permitting the replenishment of storage vesicles. Uptake 2 followed by metabolism may also play some part in the inactivation of transmitter noradrenaline, but is much more likely to be predominantly involved in the removal and inactivation of circulating catecholamines by way of the smooth muscle of blood vessels.

The two uptake processes also differ in their susceptibility to drugs. Uptake 1 is inhibited by a range of sympathomimetic amines related to noradrenaline, many of which have little effect on uptake 2. Conversely, Iversen and Salt, in a previous article (Brit. J. Pharmacol., 40, 528, 1970), have shown that certain steroids are highly effective and specific inhibitors of uptake 2 . These inhibitors include corticosterone, 17 - $\beta$-oestradiol and deoxycorticosterone, all of which occur naturally but at plasma concentrations below those required to cause an effective inhibition of uptake under normal physiological conditions. Cholesterol is present in plasma at a much higher concentration, however, and, although as described by Iversen and Salt in their present article, it is a less potent antagonist of uptake 2 than any of the steroids previously investigated, it could exert a significant inhibitory influence on the effectiveness with which catecholamines are removed from the circulation under conditions encountered physiologically.

This blockade would be more marked when the plasma cholesterol is increased, for instance as a result of a high dietary intake, and in this way cardiovascular changes leading to the development of hypertension could be brought about. 